\title{
NOTA SOBBRE OS FUNDOS DETRÍTICOS DO CIRCALITORAL INFERIOR DA PLATAFORMA CONTINENTAL BRASILEIRA AO SUL DO CABO FRIO (RJ) *
}

(Recebido em 12/9/1969)

Luiz Roberto Tommasi

Instituto Oceanográfico da Universidade de São Paulo

\begin{abstract}
SYNOPSIS
Some observations on the circalittoral benthic fauna of the region south of Cabo Frio (RJ) are presented. This fauna is similar to that of Mediterranean and Oriental Atlantic region.
\end{abstract}

\section{INTRODUÇÃO}

Como acentuou muito bem PÉRÈs (1967), as populações bênticas do circalitoral se diferenciam bàsicamente das do infralitoral por um aumento das populações sesseis animais, em detrimento das de algas. É também característico dêsses fundos, a possibilidade de apresentar o fenômeno das concreçôes produzidas por organismos secretores de calcáreos. Nesses locais, ocorrem as chamadas biocenoses coraligenas, que permitem desenvolver um substrato duro, a partir de um mole, criando assim, condições novas, não sòmente para a flora e fauna sesseis, mas também, para as espécies animais sedentárias e para as pouco vágeis (PÉRÈs, op. cit.).

$\mathrm{Na}$ presente nota, apresentamos algumas obser. vações que efetuamos durante nossos trabalhos de levantamento faunístico do circalitoral da plataforma continental ao sul do Cabo Frio (RJ). A fauna do nível bêntico dessa região é ainda, pràticamente, inex. plorada.

\section{OBSERVAÇÃO SÔBRE A FAUNA CIRCALITORAL}

Em seu trabalho sôbre os corais do circalitoral, coletados durante a viagem do "Hassler", Pourtalès (1874) assinalou a ocorrência das seguintes espécies no Brasil:

\section{1 - Axohelia (Stylophora) dumetosa Duch. $11^{\circ} 49^{\prime} \mathrm{S}-22 \mathrm{~m}$ prof.}

- Parte do material utilizado no presente trabalho, foi obtido durante as viagens do N/Oc. "Prof. W. Besnard" dentro do convênio entre o Grupo Estadual para 0 Desenvolvimento da Indústria de Pesca e o Fundo de Pesquisas do Instituto Oceanográfico da USP. Publ. n.o 292 do Inst. Ocean. da USP.
2 - Flabellum braziliense Pourtalès $11^{\circ} 49^{\prime} \mathrm{S}$ - $73 \mathrm{~m}$ prof.

3 - Madracis asperula M. Edw. \& Haine $11^{\circ} 49^{\prime} \mathrm{S}$ - $73 \mathrm{~m}$ prof.

4 - Sphenotrochus sp. $11^{\circ} 49^{\prime} \mathrm{S}-22-33 \mathrm{~m}$ prof.

5 - Bathycyathus maculatus Pourtalès ao longo de Abrolhos - $55 \mathrm{~m}$ prof.

6 - Cladocora patriarca Pourtalès ao largo de Cabo Frio $-64 \mathrm{~m}$ prof.

7 - Thecocyathus cylindraceus Pourtalès ao largo de Cabo Frio - $64 \mathrm{~m}$ prof.

Posteriormente à êsse trabalho, só conhecemos o de SQuires (1959) que assinalou, para a região da Flórida (U.S.A.), dois gêneros dêstes animais (Asterosmilia e Stenocyathus), bem como Madrepora oculata Lin., para o norte do Rio de Janeiro $\left(20^{\circ} 37^{\prime} \mathrm{S}-34^{\circ} 37^{\prime} \mathrm{W}\right)$ a $55 \mathrm{~m}$ de profundidade.

Em seu trabalho sôbre a plataforma continental dos Estados de Alagoas e Sergipe, Mabesoone \& $\mathrm{T}_{1}$. Noco (1967) encontraram corais vivos e mortos com $8-12 \mathrm{~cm}$ de tamanho. Não citam, porém a que gê. nero pertencem.

Em nossas dragagens na plataforma continental ao sul do Cabo Frio (Tabela I) temos encontrado freqüentemente, entre 120 e $200 \mathrm{~m}$ de profundidade, na altura dos Estados do Rio de Janeiro e São Paulo, e de $46 \mathrm{~m}$ em diante na do Rio Grande do Sul, um fundo arenoso, rico em fragmentos de conchas, valvas de branquiópodes e fragmentos de briozoários. 
TABELA I - Dados das estacões realizadas em fundos de circalitorais ao sul do Cabo Frio (RJ)

\begin{tabular}{|c|c|c|c|c|}
\hline \multirow[b]{2}{*}{$\begin{array}{l}\text { Estacão e } \\
\text { Embarcação }\end{array}$} & \multirow[b]{2}{*}{ Posição } & \multirow{2}{*}{$\begin{array}{l}\text { Profun- } \\
\text { didade } \\
\text { (m) }\end{array}$} & \multicolumn{2}{|c|}{ Agua de fundo } \\
\hline & & & $\begin{array}{l}\text { Tempe- } \\
\text { ratura } \\
\left({ }^{\circ} \mathrm{C}\right)\end{array}$ & $\begin{array}{l}\text { Salini- } \\
\text { dade } \\
(\%)\end{array}$ \\
\hline $\begin{array}{l}\text { D. Bentos } \\
\text { "Emilia" }\end{array}$ & $\begin{array}{l}24^{\circ} 15^{\prime} \mathrm{S} \\
44^{\circ} 00^{\prime} \mathrm{W}\end{array}$ & 180 & * & 36.53 \\
\hline $\begin{array}{l}\text { 296. Bentos } \\
\text { "Prof, W. Bes- } \\
\text { nard" }\end{array}$ & $\begin{array}{l}24^{\circ} J 2^{\prime} \mathrm{S} \\
44^{\circ} 13^{\prime} \mathrm{W}\end{array}$ & 133 & * & $=$ \\
\hline $\begin{array}{l}\text { Pesca explora- } \\
\text { tória (Pe) } \\
\text { "Prof. W. Bes- } \\
\text { nard" }\end{array}$ & $\begin{array}{l}24^{\circ} 30^{\circ} \mathrm{S} \\
44^{\circ} 54^{\prime} \mathrm{W}\end{array}$ & 125 & * & * \\
\hline $\begin{array}{l}\text { 568. Idem } \\
\text { "Prof. W. Bes- } \\
\text { nard" }\end{array}$ & $\begin{array}{l}29^{\circ} 20^{\prime} \mathrm{S} \\
48^{\circ} 57^{\prime} \mathrm{W}\end{array}$ & 78 & 17.03 & 35.90 \\
\hline $\begin{array}{l}\text { 383. Idem } \\
\text { "Prof. W. Bes- } \\
\text { nard" }\end{array}$ & $\begin{array}{l}32^{\circ} 40^{\prime} \mathrm{S} \\
51^{\circ} 36^{\prime} \mathrm{W}\end{array}$ & 46 & 15.78 & 33.56 \\
\hline
\end{tabular}

* Sem informaçōes.

Nesse fundo coletamos numerosas conchas de Cavolina sp., Columella sp., Conus sp., Murex sp., Antalis sp., Veneridae, Chlamys sp., Haliotis sp., ascideas (Molgula, Pyuridae, Styelidae), braquiópodes vivos do gênero Tebratula, crustáceos dos gêneros Parthenope sp., Munida sp., Macrocoeloma sp., Euprognatha sp., Portunus sp., numerosos ofiuróides como: (Ophiothrix rathbuni Ludwig, Ophiomisidium pulchellum (W. Thompson), Ophiacantha vivipara Ljg.), os equinóides Stylocidaris affinis, Philippi, Echinociamus grandiporus Mortensen, o asteróide Leptasterias hartii Rathbun, o crinóide Neocomatella pulchella (Pourtalès), peixes demersais como Dules auriga Cuv., Prionotus beanii Good e diversas espécies de corais. Destas, as principais, estão abaixo relacionadas e foram gentilmente classificadas por Jacques Laborel:

1 - Cladocora arbuscula (Lesseur) (Figs. 5 e 6)

2 - Dasmosmilia lymani (Pourtalès) (Fig. 2)

3 - Deltocyathus italicus M. Edw. \& Haine (Figs. 5 e 7)

4 - Madracis mirabilis (Duch. \& Michellotti) (Fig. 1)

5 - Trochocyathus sp. (Fig. 3)

Além dessas espécies, obtivemos com freqüência menor: Primnoella af. delicatissima Kiib. (Fig. 4) e Ellisella sp. (Fig. 6).
Nas estações 568 e 383 (Mapa 1) foram frequentes numerosas gorgônias, bem como o ofiuróide Astrocyclus caecilia (Fig. 8).

Esses fundos correspondem aos fundos detríticos do bordo da plataforma (fonds détritiques du large) da região inferior do circalitoral, assinalados por PÉRÈs (1967) no Mediterrâneo e ao largo da costa francesa do Atlântico, e por Longhurst (1958) ao largo da Guiné e do Senegal, nos quais ocorrem vários gêneros idênticos aos da plataforma ao sul de Cabo Frio, ou seja: Ophiothrix, Ophiacantha, Dentalium. etc.. O gênero Leptometra (crinóide), ocorrente naquelas regiões, é aqui ecològicamente substituído por Neocomatella.

Os fundos detríticos do bordo da plataforma, na região ao sul de Cabo Frio, estão sujeitos a uma massa caracterizada por apresentar salinidade entre $35-36^{\circ} \%$ e temperatura de $10-20^{\circ} \mathrm{C}$ e que foi denominada por Emilsson (1959) "água de plataforma". taforma".

No Mapa 1 são apresentadas 5 estações nas quais encontramos o fundo detrítico com corais de profundidade. Nas Figuras 5-7, apresentamos alguns dos animais mais característicos dessas estações.

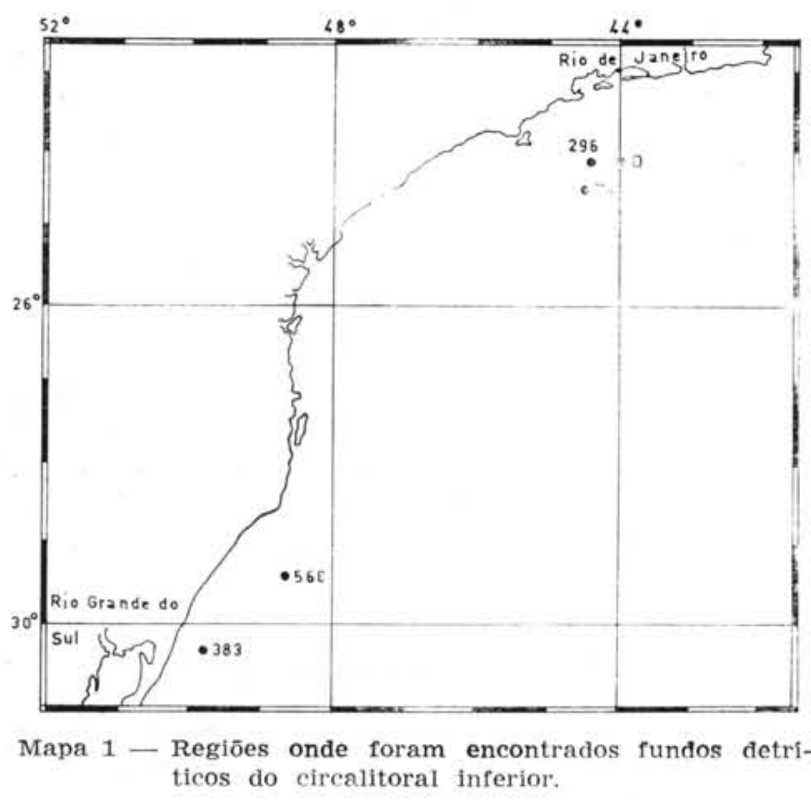

Dado o grande desconhecimento da fauna do circalitoral da região presentemente estudada, acreditamos ser útil divulgar as fotografias das Figuras 1 a 4 que foram efetuadas por Jacques Laborel e as 5 a 8 efetuadas pelo Sr. Renato Herz da Secção de Fotocinematografia e Desenho da Divisão de Informação e Documentação Científica do Instituto Oceanográfico da U.S.P. 


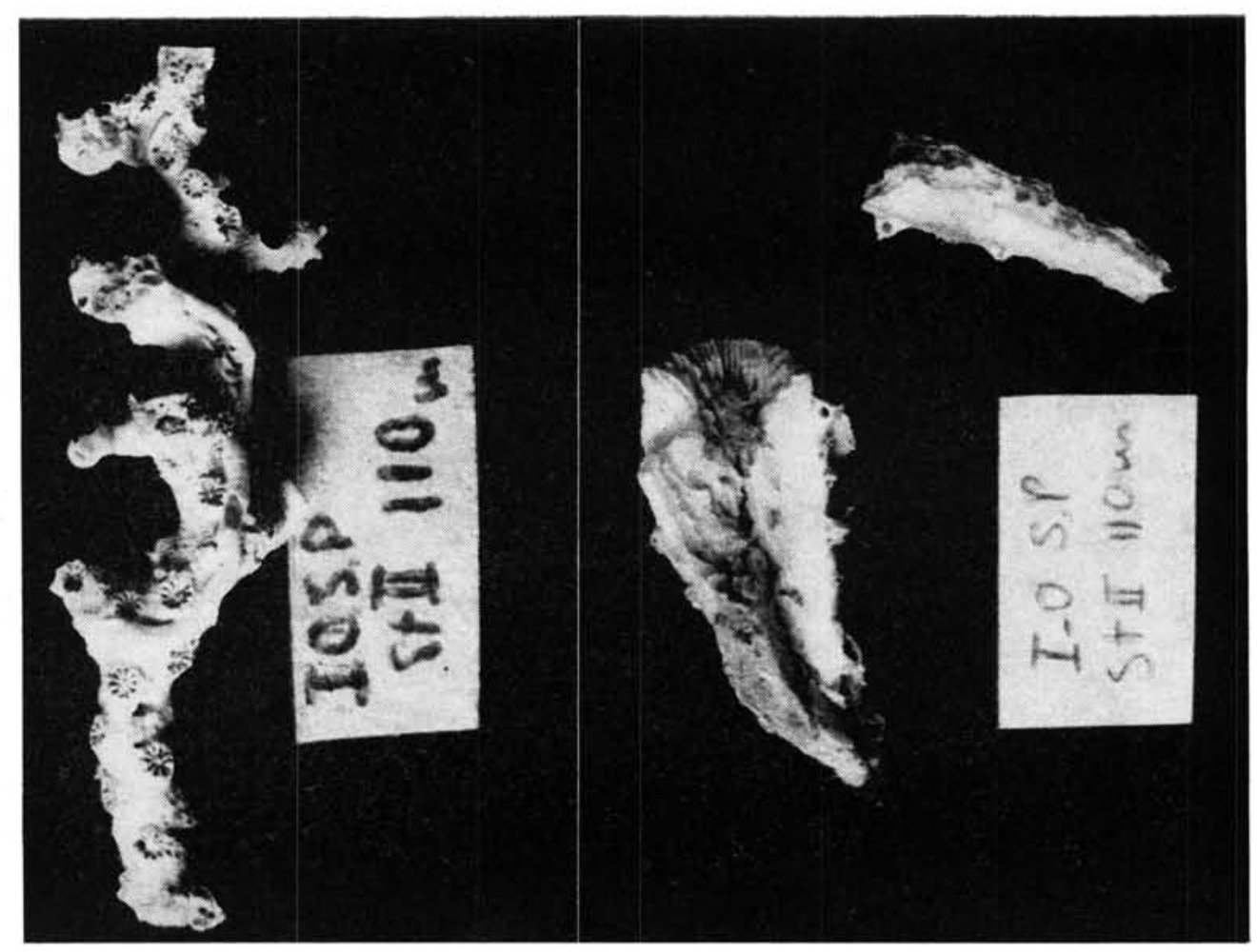

Fig. 1-Madracis mirabilis (Duch. \& Michellotti).

Fig. 2 - Dasmosmilia lymani (Pourtaiès).

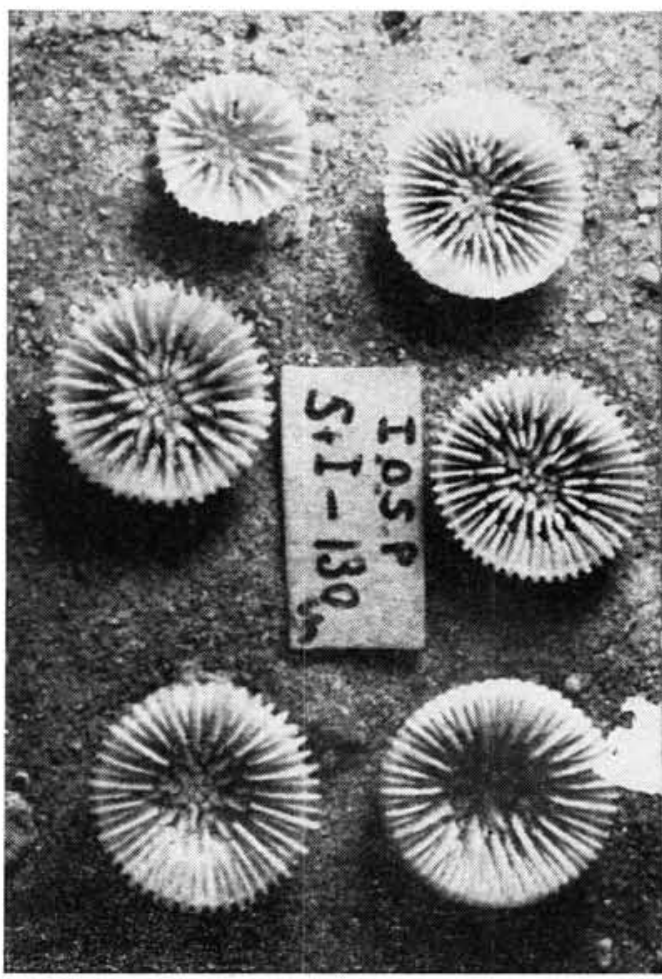

Fig. 3 - Trochocyathus sp.

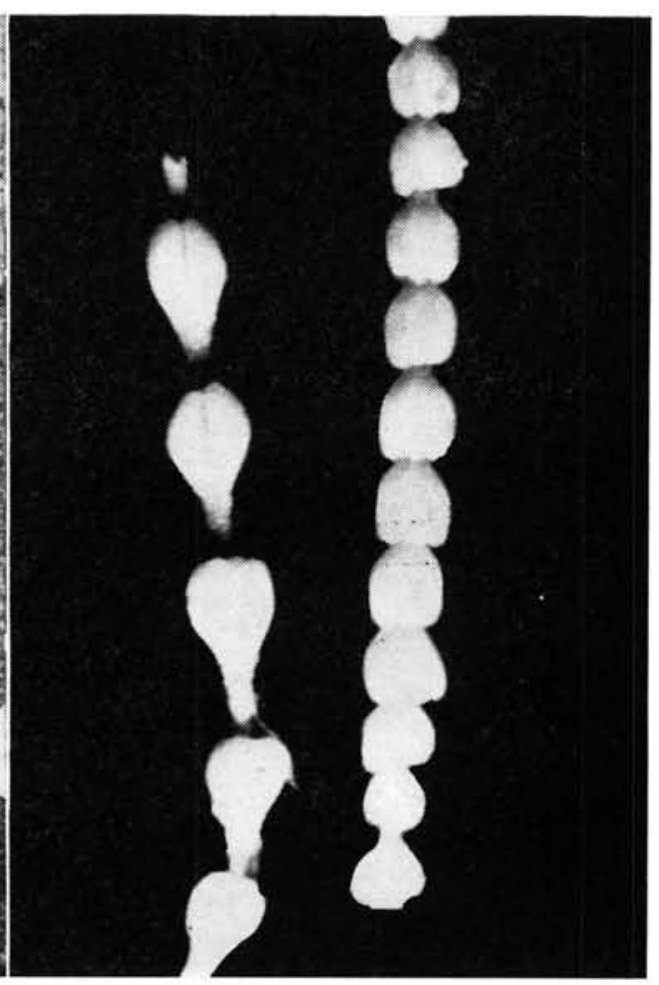

Fig. 4 - Primnoella af. delicatissima Kilb. 


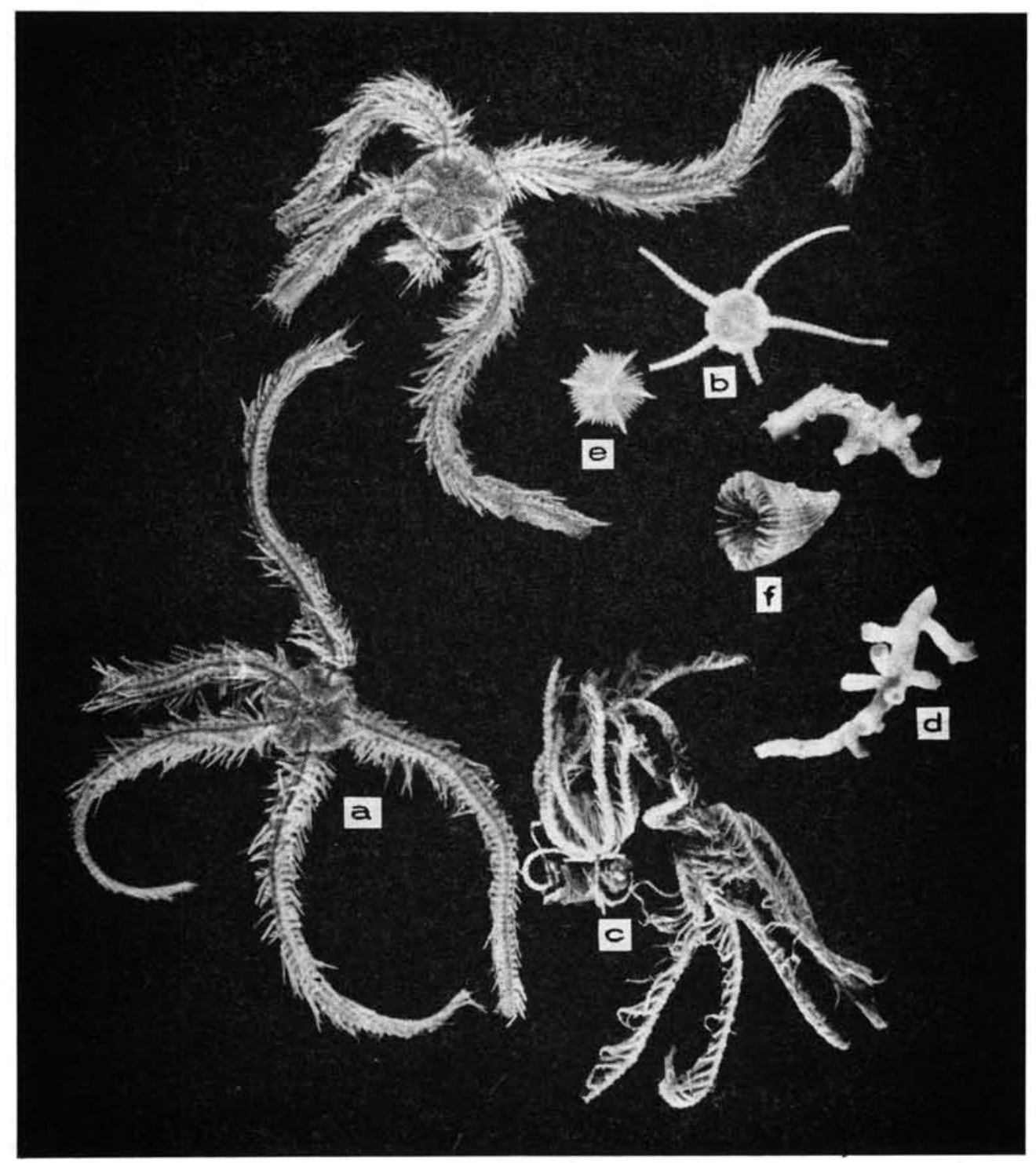

Fig. 5 - Animais caracteristicos das estacões D e 296:

a - Ophiothrix rathbuni

b - Ophiomisidium pulchellum

c - Neocomatella pulchella

d - Cladocora arbuscula

e - Deltocyathus italicus

$\mathrm{f}$ - Trochocyathus sp 


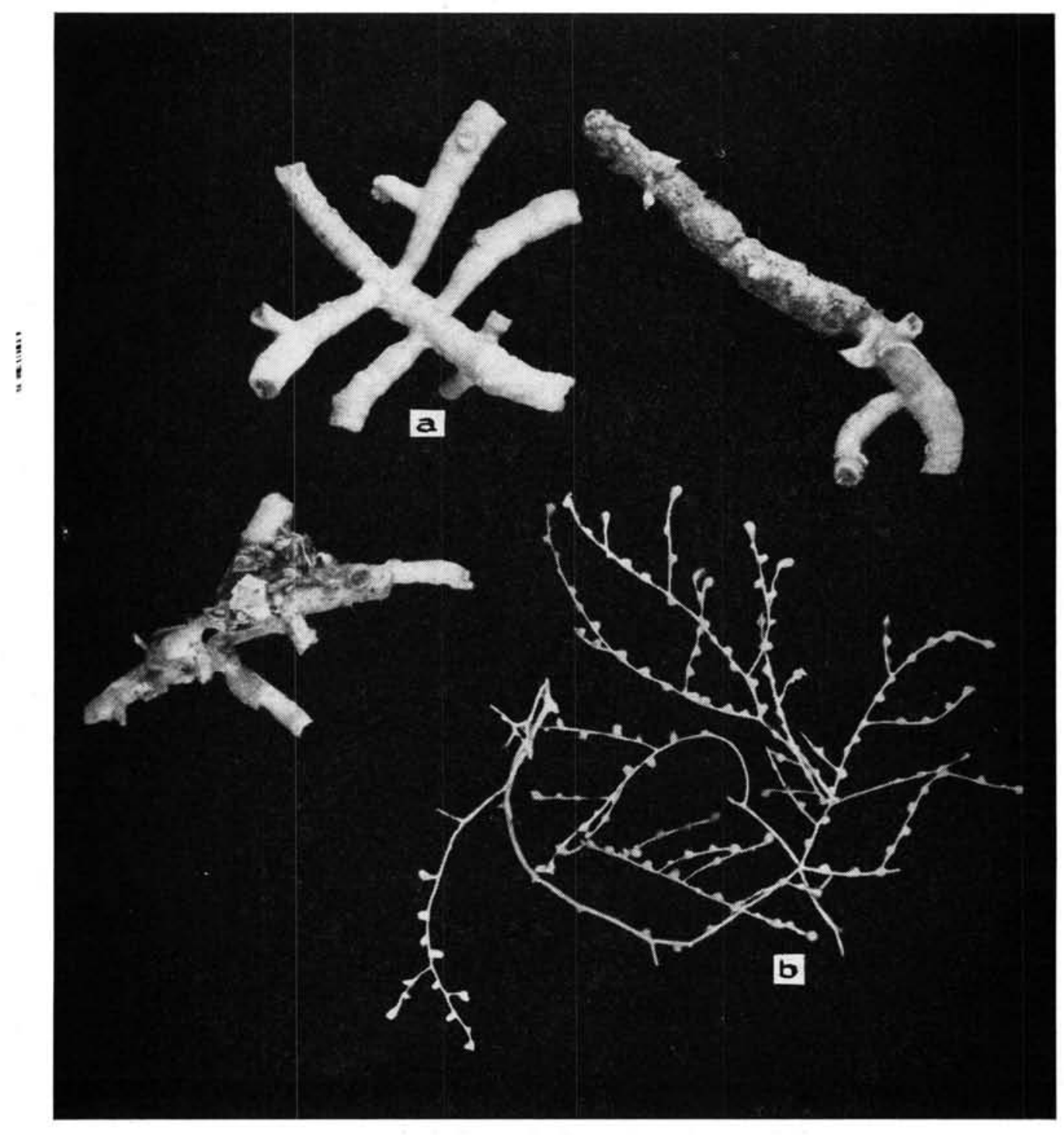

Fig. 6 - Animais característicos da estaçâo Pe a - Cladocora arbuscula

b - Ellisella sp. 


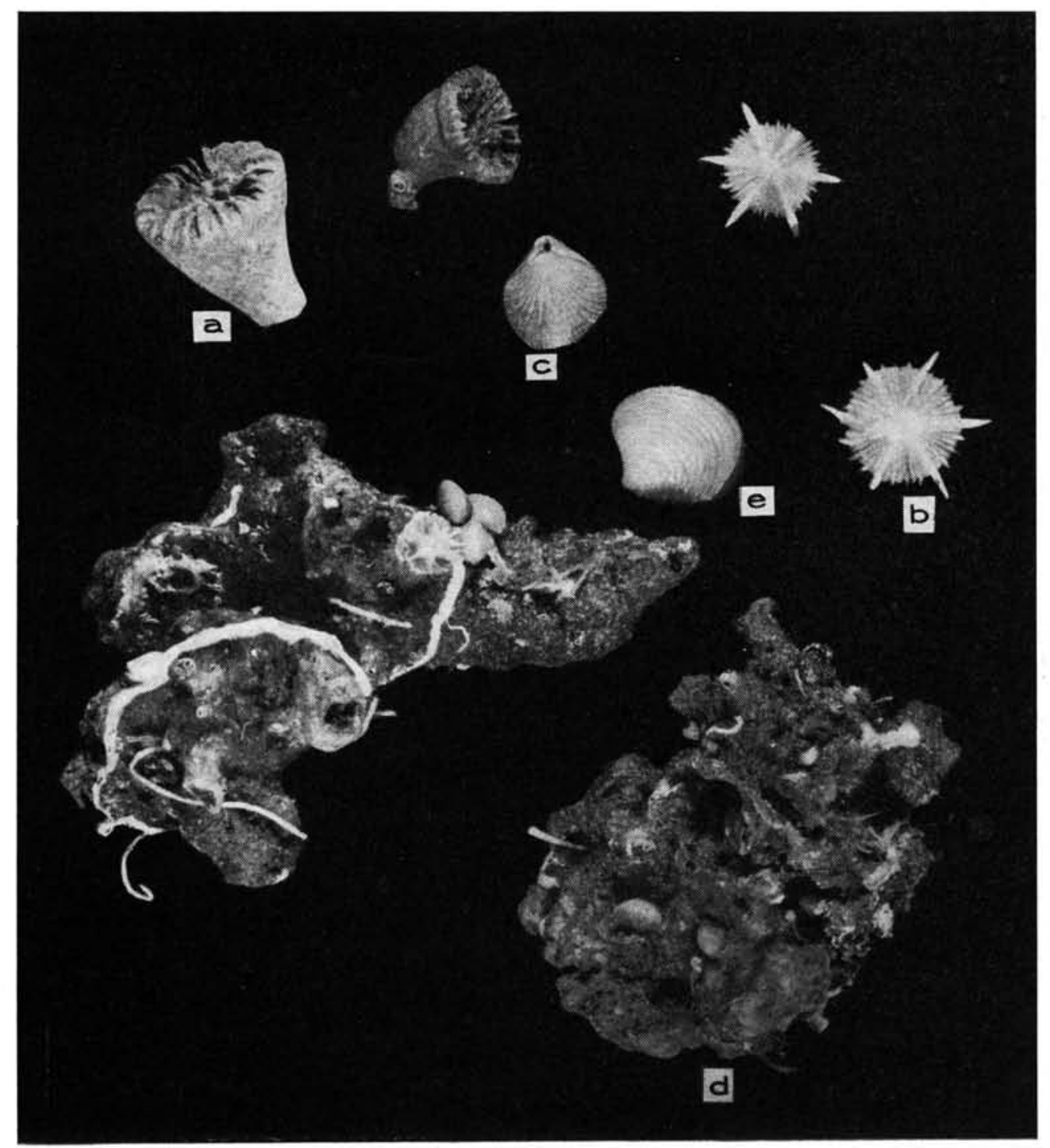

Fig. 7 - Animais caracteristicos das estacões 568 e 383:

a - Trochocyathus sp. (?)

b - Deltocyathus italicus

c - braquiópode (espécie a)

d - pedra com braquiópodes (espécie b), coral, tubos de poliqueta e esponja

e - lamelibrånquio 


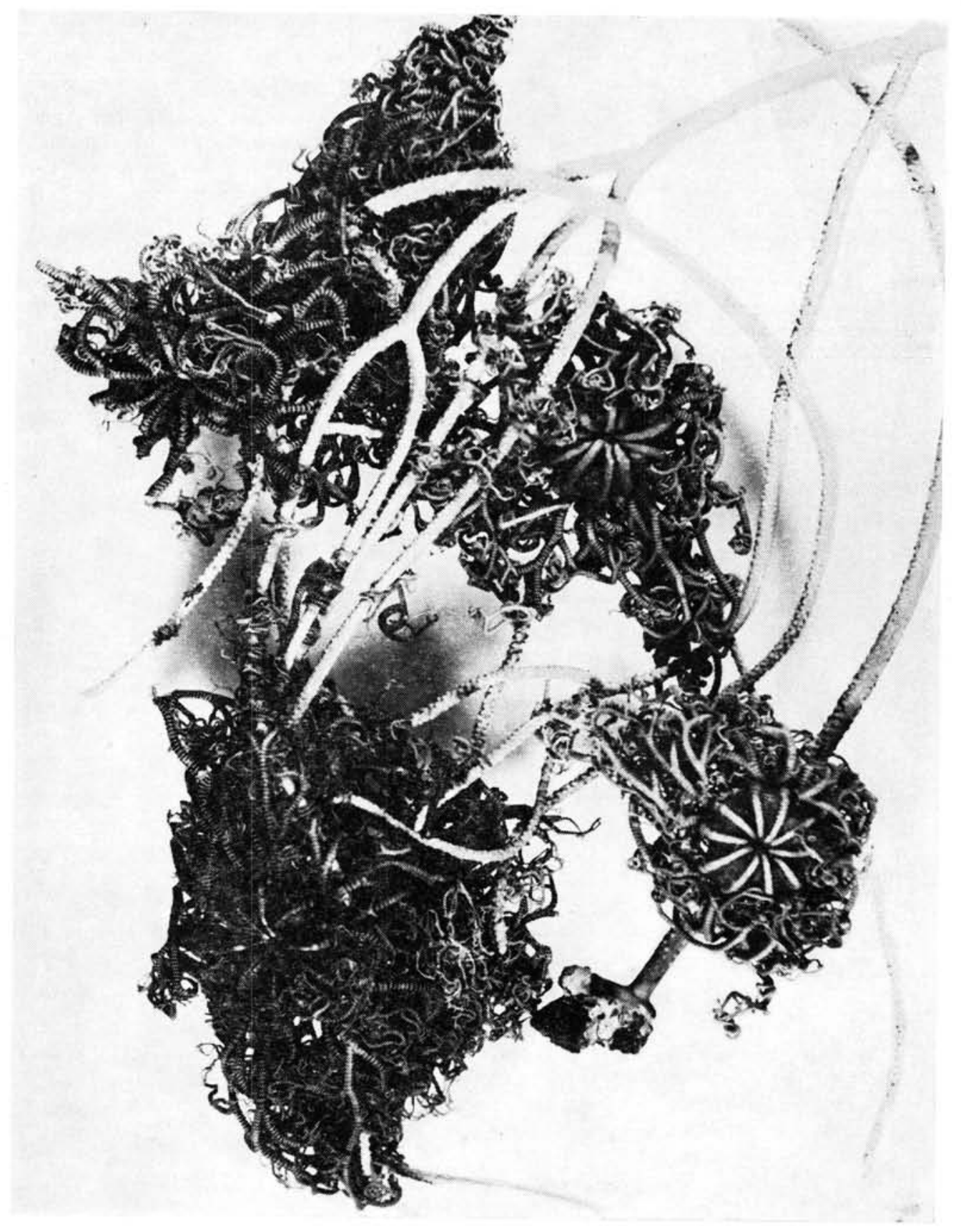

Fig. 8-Astrocyclus caecilia sôbre gorgônia. 


\section{SUMMARY}

In the circalittoral of south of Cabo Frio region $\left(23^{\circ} \mathrm{S}\right)$ we found a rich fauna in a calcareous coraline bottom. The most common species are the corals, Cladocora arbuscula (Less.), Dasmosmilia lymani (Poust.), Deltocyathus italicus M. Edw. \& Haine, Madracis mirabilis (Duch. \& Michellotti) and Trochocyathus sp, the echiroderms Stylocidaris affinis Phil., Echinociamus grandiporus Mrtsn., Neocomatella pulchella (Pourt.). There are numerous shells of $\mathrm{Ca}$ volina sp. and Columella sp.

\section{B I B L I O G R A F I A}

EMILSSON, I.

1959. Alguns aspectos físicos e quimicos das águas marinhas brasileiras. Cienc, Cult. $\mathrm{S}$ Paulo, vol. $11, \mathrm{n}^{\circ}{ }^{2}$, p. $44-54$.

LONGHURST, A. R.

1958. An ecological survey of the west African marine benthos. Col. office, Fish. Publ., vol. 11, 102 p.
MaBesoone, J. M. \& Tinoco, I. M.

1967. Shelf off Alagoas and Sergipe. Trabhs Inst. oceanogr. Univ. Fed. Pe., vol. 7/8, p. $151-186$.

PÉRÈS, J. M.

1967. Les biocoenoses benthiques dans le système phytal. Recl. Trav. Stn. mar. Endoume, vol. 42, n. $^{\circ} 58$, p. $3-113$

Pourtalès, L. F. de

1874. Deep sea corals. In: Agassiz, A., Zoological results of the "Hassler" expedition. Mem. Mus. comp. Zool. Harv., vol, 4, p. 27-50.

SQUIRES, D. F.

1959. Deep sea corals collected by the Lamont Geological Observatory. I. Atlantic corals. Am. Mus, Novit., n.o 1965, 42 p. 